\title{
Aggregation of a Class of Large-scale, Interconnected, Nonlinear Dynamical Systems
}

\author{
SWAROOP DARBHA* and K.R.RAJAGOPAL ${ }^{\dagger}$ \\ Department of Mechanical Engineering, Texas A\&M University, \\ College Station, TX - 77843-3123
}

(Received 3 January 2001; In final form 12 February 2001)

In this paper, the authors consider the issue of the construction of a meaningful average for a collection of nonlinear dynamical systems. Such a collection of dynamical systems may or may not have well defined ensemble averages as the existence of ensemble averages is predicated on the specification of appropriate initial conditions. A meaningful "average" dynamical system can represent the macroscopic behavior of the collection of systems and allow us to infer the behavior of such systems on an average. They can also prove to be very attractive from a computational perspective. An advantage to the construction of the meaningful average is that it involves integrating a nonlinear differential equation, of the same order as that of any member in the collection. An average dynamical system can be used in the analysis and design of hierarchical systems, and will allow one to capture approximately the response of any member of the collection.

Keywords: Aggregation; String of dynamical systems; String stability; Average dynamical system; Averaging

\section{INTRODUCTION}

Aggregation of large scale systems is important for two reasons firstly, it provides clarity in the analysis and design of large scale systems by providing a simpler but macroscopic picture of the system; secondly, since the evolution of aggregates can be approximated by

\footnotetext{
*Corresponding author. Tel.: 979-862-2238, e-mail: dswaroop@mengr.tamu.edu

†e-mail: krajagopal@mengr.tamu.edu
} 
"reduced order" differential equations, the burden of computing and obtaining a macroscopic picture of a large scale system is significantly reduced.

In this paper, we are concerned with averaging a class of homogeneous collection of dynamical systems. In a homogeneous collection, the structure of the dynamics of the systems and the interactions amongst systems is identical. Therefore, averaging the states of the collection of systems makes sense.

A formal definition of aggregation and restriction for finite dimensional systems is given in [7]. In [22], we developed a framework for constructing meaningful averages for a countable collection of LTI systems.

This research is inspired by our prior work on the modeling of traffic flow behavior as a function of the adaptive cruise control designs used by the constituent vehicles in the traffic [21, 22]. The problem is that of determining the evolution equations for the density and speed of traffic when vehicles in traffic automatically change their acceleration in response to changes in their following distance and in their rate of change of the following distance. In such a scenario, the average following distance of vehicles is inversely proportional to the density of traffic and the average speed of vehicles is a good macroscopic representation of the traffic speed. Such a macroscopic description of traffic flow can be utilized to examine the propagation of shock waves in traffic and consequently, to evaluate different adaptive cruise control systems.

An important issue in aggregation is the sensitivity of the aggregate to the size of the collection. For example, if the average behavior of a traffic were to be significantly different with a million and one vehicles on the highway as opposed to a million vehicles, then that behavior cannot really be thought of as a macroscopic representation of the underlying microscopic behavior of traffic. It is this issue that brings forth the limitations of an ensemble average.

We envision the presented research to have applications in controlling formations. In [17], the authors consider the problem of controlling the average position of a platoon of underwater vehicles; similarly, in [23], the authors consider a problem of controlling the average position of air vehicles flying in a formation.

If the aggregate of initial conditions of the collection of systems cannot be well defined, it is quite likely that the aggregate may not be a 
meaningful macroscopic representation of the collection. This can be the case if the initial conditions form a geometric sequence with a common ratio greater than unity. It is, in this sense, an ensemble average is limited in its ability to be a meaningful macroscopic aggregate.

In some instances, the structure of the interactions may be such that a meaningful macroscopic aggregate may not exist. For example, in automatic vehicle following systems, collective or string instability can occur, although, individually, each vehicle can track its predecessor with a bounded error. However, the bound keeps increasing with the vehicle index, in some cases, it amplifies geometrically, when there is string instability. In such a collection, the computation of an ensemble average of following distances is not really indicative of the density of traffic; in fact, it is not a meaningful aggregate.

In order to circumvent the need to verify if the ensemble average of the initial conditions is sensitive to the size of the collection, we introduce the notion of a meaningful average. A meaningful average is an ensemble average for meaningful initial conditions and is dependent solely on the structure of the interactions. We refer to $w(t)$ as a meaningful average for a countable collection of systems, if the initial conditions of all the systems in the collection start close to $w(0)$, then the state of every system in the collection is close to $w(t)$. In addition, it is required that if the average of the initial conditions is $w(0)$, then the average of the states is $w(t)$. What we seek in the following sections, is a "reduced order" differential equation that describes the evolution of $w(t)$.

\section{PROBLEM STATEMENT}

In this paper, we will consider the following class of interconnected, nonlinear systems with finite interactions:

$$
\begin{gathered}
\dot{x}_{i}=f_{i}\left(x_{i}, x_{i-1}, \ldots, x_{i-r+1}, u\right), \quad i=1,2, \ldots, \\
\text { and } \quad x_{i} \equiv x_{1}, \quad \forall i \leq 1 .
\end{gathered}
$$

Here, $x_{\mathrm{i}}(t) \in \Re^{n}, r$ is a fixed, finite, positive integer, The vector fields, $f_{\mathrm{i}}$ are assumed to be sufficiently differentiable; in addition, it will 
be assumed that solution to this countable system of differential equations exist and is unique. The term, $u(t) \in \mathcal{R}^{m}$, is piece-wise continuous in $t$ and represents the reference information available to each system; it will be assumed to be bounded. The interconnected system described by the above equations will be referred to as a string. The following notation is used in this paper: If $a$ is a vector in $\mathcal{R}^{n},\|a\|$ represents its Euclidean norm. If for every $t, b(t) \in \mathcal{R}^{n}$, $\|b(t)\|_{p}$ represents $\left\{\int_{0}^{\infty}\|b(t)\|^{p} d t\right\}^{(1 / p)}$. Notice that the definition of norm presented here involves integration over time.

The following notion of stability of a solution $[4,20]$ to the above system of differential equations, also referred to as string stability, will be used in the rest of the paper:

Definition $2.1\left(\mathcal{L}_{p}\right.$ stability) Let $\left\{x_{i}(0)\right\}$ and $\left\{\hat{x}_{i}(0)\right\}$ be two sets of initial conditions and let $\left\{x_{i}(t)\right\}$ and $\left\{\hat{x}_{i}(t)\right\}$ be the corresponding solutions. Given $\varepsilon>0$ if there exists a $\delta>0$ such that

$$
\sup _{i}\left\|x_{i o}-\hat{x}_{i o}\right\|<\delta \Rightarrow \sup _{i}\left\|x_{i}(t)-\hat{x}_{i}(t)\right\|_{p}<\varepsilon,
$$

then the solution $\left\{x_{i}(t)\right\}$ is defined to be $\mathcal{L}_{p}$ stable.

Definition 2.2 (Spatial Asymptotic $\mathcal{L}_{p}$ stability) Let $\left\{x_{i}(0)\right\}$ and $\left\{\hat{x}_{i}(0)\right\}$ be two sets of initial conditions and let $\left\{x_{i}(t)\right\}$ and $\left\{\hat{x}_{i}(t)\right\}$ be the corresponding solutions. Then, $\left\{x_{i}(t)\right\}$ is an asymptotically $\mathcal{L}_{p}$ stable solution, if it is an $\mathcal{L}_{p}$ stable solution and if

$$
\lim _{k \rightarrow \infty}\left\|x_{k}(0)-\hat{x}_{k}(0)\right\|=0 \Rightarrow \lim _{k \rightarrow \infty}\left\|x_{k}(t)-\hat{x}_{k}(t)\right\|_{p}=0 .
$$

The notion of stability presented here implies continuous dependence of solutions on the initial condition. From the definition of stability, and from the smoothness of solutions, it is clear that $\lim _{t \rightarrow \infty} \sup _{k}\left\|x_{k}(t)-\hat{x}_{k}(t)\right\|=0$. Otherwise, $\left\|x_{k}(t)-\hat{x}_{k}(t)\right\|_{p}$ will be unbounded for some $k$. Only in the case of $\mathcal{L}_{\infty}$ stability is there a need to define an asymptotic (temporal) stability of the solution and this definition is given in [19]. The notion of asymptotic stability presented here is related to the attenuation of state with respect to the system index, and not with respect to the time.

Given any time $t$, a "pointwise in time" or partial state average, or average in the sense of Cesaro, $\bar{x}_{N}(t)$, is defined as: 
$\bar{x}_{N}(t):=(1 / N) \sum_{1}^{N} x_{i}(t)$. The partial state average is well defined, since the solution of the above equation is well defined for all finite $i$. We define an "ensemble" state average at a time $t$ as

$$
\bar{x}(t):=\lim _{N \rightarrow \infty} \bar{x}_{N}(t)
$$

whenever the limit on the right hand side of the above equation exists.

The following countable collection of decoupled first order systems [22] illustrates the need for choosing a meaningful set of initial conditions for aggregation:

$$
\dot{x}_{i}=-x_{i}, \quad x_{i}(0)=(-1)^{i}(2 i-1), \quad i=1,2, \ldots
$$

For this problem, the partial average exists for every $t$. However, the ensemble average is not defined for any $t$, because of the manner in which the initial conditions are defined. Suppose the initial condition is such that $\lim _{N \rightarrow \infty}(1 / N) \sum_{1}^{N} x_{k}(0)$ exists, then the ensemble average, $\bar{x}(t)$ is given by $\lim _{N \rightarrow \infty}(1 / N) \sum_{1}^{N} x_{k}(0) e^{-t}$. As we have seen in the above example, the existence of an ensemble average is intimately related to the initial condition. We seek to define an average that is independent of the initial condition, but, one that is dependent on the structure of the system. To this end, we ask the following question: Suppose a candidate function $w(t)$ were given. If the initial condition of every member of the system is close to $w(0)$, will the resulting partial state average stay close to $w(t)$ ? If, in addition, the initial conditions are such that the ensemble average of the initial conditions equals $w(0)$, is the resulting trace ${ }^{1}$ of ensemble average equal to the given candidate function, $w(t)$, at almost all instants of time?

Definition 2.3 (Meaningful Average in the strong sense) $w(t)$ is a meaningful $\mathcal{L}_{p}$ average in the strong sense, if the following two conditions hold:

(1) Given $\varepsilon>0$, there exists a $\delta>0$ such that for all solutions, $\left\{x_{k}(t)\right\}$,

$$
\sup _{k}\left\|x_{k}(0)-w(0)\right\|<\delta \Rightarrow \sup _{k}\left\|x_{k}(t)-w(t)\right\|_{p}<\varepsilon,
$$

\footnotetext{
${ }^{1}$ We use trace as in the context of a signal and not in the context of a linear operator.
} 
(2) For all solutions, $\left\{x_{k}(t)\right\}$, such that $\sup _{k}\left\|x_{k}(0)-w(0)\right\|$ is bounded, and

$$
\begin{aligned}
\left\|\frac{1}{N} \sum_{1}^{N} x_{k}(0)-w(0)\right\| & \leq \frac{M}{N^{\alpha}}, \quad \text { for some } M>0, \alpha>1 \\
& \Rightarrow\left\|\frac{1}{N} \sum_{1}^{N} x_{k}(t)-w(t)\right\|_{p} \leq \frac{\bar{M}}{N^{\beta}}, \quad \text { for some } \bar{M}, \beta>0 .
\end{aligned}
$$

Any function of the form $A e^{-t}$ is a meaningful average for the previously considered collection of decoupled first order systems.

Consider the following countable collection of coupled first order systems [22]:

$$
\begin{gathered}
\dot{x}_{i}=-x_{i}+2 x_{i-1}+u, \quad i=1,2, \ldots ; \\
x_{i}=0, \quad \forall i \leq 0, u=1.0 ; \quad x_{i}(0)=0, \forall i ; \\
y_{i}=x_{i} .
\end{gathered}
$$

Denoting the Laplace Transform of $x_{i}(t)$ by $X_{i}(s)$ and taking the Laplace Transformation of both sides of the above differential equation, we get:

$$
X_{i}(s)=\frac{2}{s+1} X_{i-1}(s)+\frac{1}{s(s+1)} .
$$

Then, by an inductive argument, one obtains:

$$
X_{k}(s)=\sum_{j=1}^{k}\left(\frac{2}{s+1}\right)^{k-j} \frac{1}{s(s+1)}
$$

Application of the final value theorem indicates that $\lim _{t \rightarrow \infty} x_{k}(t)=$ $2^{k}-1$. This indicates that $\lim _{t \rightarrow \infty} \bar{x}_{N}(t)=\left(2^{N+1}-N-1\right) / N$. Next, consider

$$
X_{k}(s)-X_{k-1}(s)=\frac{2^{k-1}}{s(s+1)^{k}} .
$$

The right hand side of the above equation can be written as the Laplace transform of the convolution of functions that are nonnegative at every instant of time. Therefore, $x_{k}(t)-x_{k-1}(t) \geq 0$. This implies that given any $t$, the sequence $\left\{x_{k}(t)\right\}$ is monotonically 
increasing. Since $\lim _{t \rightarrow \infty} \bar{x}_{N}=\left(2^{N+1}-N-1\right) / N$, given any $M>0$ there exists a $N^{*}>0$ such that $\lim _{t \rightarrow \infty} \bar{x}_{N}>M, \forall N \geq N^{*}$. In particular, $\lim _{t \rightarrow \infty} \bar{x}_{N^{*}}>M$. Therefore, given any $\varepsilon>0$, there exists a $t_{N^{*}}$ such that $\bar{x}_{N^{*}}>M-\varepsilon$ for all $t>t_{N^{*}}$. Since, at any $t$, the sequence $\left\{x_{k}(t)\right\}$ is monotonic, it follows that for $\bar{x}_{N}(t)>M-\varepsilon$ for all $t>t_{N^{*}}$ and for all $N>N^{*}$. Therefore, $\lim _{t \rightarrow \infty} \lim _{N \rightarrow \infty} \bar{x}_{N}(t)$ is unbounded.

In this problem, $\lim _{N \rightarrow \infty} \bar{x}_{N}(0)=0$. While $x_{i}(t), i=1,2, \ldots, N$ is always bounded for all finite $N$, the limit of the sequence of partial averages, even if it exists, is always unbounded. A meaningful average does not exist for this system.

In the first example, $\lim _{n \rightarrow \infty} \bar{x}_{n}(t)$ is not defined, whereas in second example, there is a problem of instability of the string, i.e., value of the state of the $i$-th system geometrically amplifies with the spatial index $i$. These are two important issues that determine the calculation of an average or an aggregate solution for a collection of dynamical systems.

In this paper, we are interested in constructing meaningful averages, if at all possible; in cases, where it may not be easy, we will atleast seek an approximation for ensemble average for a given initial condition.

\section{MAIN RESULTS}

Our first result concerns the following string of equations:

$$
\dot{x}_{i}=f\left(x_{i}, x_{i-1}, \ldots, x_{i-r+1}, u(t)\right), \quad x_{j}(t) \equiv x_{1}(t), \forall j \leq 1, i=1,2, \ldots
$$

Let $w(t)$ satisfy the following differential equation:

$$
\dot{w}=f(w, w, \ldots, w, u) .
$$

We will show that any solution of the above differential equation will be a meaningful average for the given collection of interconnected systems in the strong sense, provided $f$ satisfies certain conditions. To this end, we will make the following assumptions:

(1) The function, $f$ is Lipschitz, i.e., there exist $l_{1}, \ldots, l_{r}$ such that

$$
\begin{aligned}
\left\|f\left(x_{1}, x_{2}, \ldots, x_{r}, u\right)-f\left(y_{1}, y_{2}, \ldots, y_{r}, u\right)\right\| \\
\leq l_{1}\left\|x_{1}-y_{1}\right\|+l_{2}\left\|x_{2}-y_{2}\right\|+\cdots+l_{r}\left\|x_{r}-y_{r}\right\| .
\end{aligned}
$$

(2) The zero solution of the differential equation,

$$
\dot{e}=f(e+w, e+w, \ldots, e+w, u)-f(w, w, \ldots, w, u),
$$


is exponentially stable. In other words, there exists a Lyapunov function, $V(e, t)$ such that

$$
\begin{gathered}
\alpha_{1}\|e\|^{2} \leq V(e, t) \leq \alpha_{2}\|e\|^{2}, \\
\frac{\partial V}{\partial t}+\frac{\partial V}{\partial e}[f(e+w, \ldots, e+w, u)-f(w, w, \ldots, w, u)] \leq-\alpha_{3}\|e\|^{2}, \\
\left\|\frac{\partial V}{\partial e}\right\| \leq \alpha_{4}\|e\|,
\end{gathered}
$$

for some $\alpha_{1}, \alpha_{2}, \alpha_{3}, \alpha_{4}>0$.

(3) In addition, it will be assumed that the interactions between the systems are sufficiently weak, i.e., that $\sum_{2}^{r} l_{j}<\bar{l}$, where $\bar{l}$ will be defined later.

Theorem 3.1 (Computation of a meaningful average) Under the assumptions given above, $w(t)$ is a meaningful average in the strong sense if and only if it satisfies

$$
\dot{w}=f(w, w, \ldots, w, u) .
$$

Proof First, we will show that if $w(t)$ is a solution of $\dot{w}=f(w, w, \ldots$, $w, u)$, then $w(t)$ is a meaningful average.

Let us define $e_{k}(t):=x_{k}(t)-w(t)$. Then,

$$
\begin{aligned}
\dot{e}_{k}= & f\left(e_{k}+w, e_{k-1}+w, \ldots, e_{k-r+1}+w, u\right) \\
& -f(w, w, \ldots, w, u), \quad \text { with } \quad e_{i} \equiv e_{1} \quad \text { if } i \leq 1 .
\end{aligned}
$$

Let us define $V_{k}:=V\left(e_{k}, t\right)$. Therefore,

$$
\begin{aligned}
\dot{V}_{k}= & \frac{\partial V_{k}}{\partial t}+\frac{\partial V_{k}}{\partial e_{k}}\left[f\left(e_{k}+w, e_{k-1}+w, \ldots, e_{k-r+1}+w, u\right)-f(w, w, \ldots, w, u)\right] \\
=\frac{\partial V_{k}}{\partial t}+\frac{\partial V_{k}}{\partial e_{k}}\left[f\left(e_{k}+w, e_{k-1}+w, \ldots, e_{k-r+1}+w, u\right)\right. & \quad f\left(e_{k}+w, e_{k}+w, \ldots, e_{k}+w, u\right) \\
& \quad f\left(e_{k}+w, e_{k}+w, \ldots, e_{k}+w, u\right) \\
& \quad-f(w, w, \ldots, w, u)] \\
\leq- & \alpha_{3}\left\|e_{k}\right\|^{2}+\alpha_{4}\left\|e_{k}\right\|\left(l_{2}\left\|e_{k}-e_{k-1}\right\|+\cdots+l_{r}\left\|e_{k}-e_{k-r+1}\right\|\right) \\
\leq- & \bar{\alpha}_{1} V_{k}+\sum_{2}^{r} \bar{\alpha}_{j} V_{k-j+1}
\end{aligned}
$$

where $\bar{\alpha}_{1}=\left(\alpha_{3}-(3 / 2) \alpha_{4} \sum_{2}^{r} l_{j}\right) / \alpha_{2}$ and $\bar{\alpha}_{j}=\left(\alpha_{4} / 2 \alpha_{1}\right) l_{j}, j=2, \ldots, r$. 
We will say that the interconnections are weak if $\bar{l}=\left(2 \alpha_{3} \alpha_{1} /\right.$ $\left.\alpha_{4}\left(3 \alpha_{1}+\alpha_{2}\right)\right)$, and we will assume that it is the case here.

If the interconnections are weak, it follows that $\bar{\alpha}_{1}>\sum_{2}^{r} \bar{\alpha}_{j}$.

CLAIM If the sequence of initial condition errors, $\left\{e_{k}(0)\right\}$, is bounded, then

$$
\begin{aligned}
& \sup _{k} \sup _{t \geq 0}\left\|e_{k}(t)\right\| \leq \sqrt{\frac{\alpha_{2} \bar{\alpha}_{1}}{\alpha_{1}\left(\bar{\alpha}_{1}-\sum_{2}^{r} \bar{\alpha}_{j}\right)}} \sup _{k}\left\|e_{k}(0)\right\|, \\
& \sup _{k}\left\|e_{k}(t)\right\|_{p} \leq \sqrt{\frac{\alpha_{2} \bar{\alpha}_{1}}{\alpha_{1}\left(\bar{\alpha}_{1}-\sum_{2}^{r} \bar{\alpha}_{j}\right)}} \sup _{k}\left\|e_{k}(0)\right\|
\end{aligned}
$$

Proof of Claim The central idea of the proof is the same as that in [20]. If $\left\{e_{k}(0)\right\}$ is a bounded sequence, then $\left\{V_{k}(0)\right\}$ is a bounded sequence.

$$
\begin{gathered}
\dot{V}_{k}=-\bar{\alpha}_{1} V_{k}+\sum_{2}^{r} \bar{\alpha}_{j} V_{k-j+1} \\
\Rightarrow V_{k}(t) \leq e^{-\bar{\alpha}_{1} t} V_{k}(0)+\sum_{2}^{r} \bar{\alpha}_{j} \int_{0}^{t} e^{-\alpha_{1}(t-\tau)} V_{k-j+1}(\tau) d \tau \\
\Rightarrow\left\|V_{k}(t)\right\|_{p} \leq V_{k}(0)+\sum_{2}^{r} \frac{\bar{\alpha}_{j}}{\bar{\alpha}_{1}}\left\|V_{k-j+1}(t)\right\|_{p} \\
\quad \text { and }\left\|V_{k}\right\|_{\infty} \leq V_{k}(0)+\sum_{2}^{r} \frac{\bar{\alpha}_{j}}{\bar{\alpha}_{1}}\left\|V_{k-j+1}\right\|_{\infty} .
\end{gathered}
$$

Since $\sum_{2}^{r} \bar{\alpha}_{j}<\bar{\alpha}_{1}$, it follows that

$$
\begin{aligned}
\sup _{k} \sup _{t \geq 0}\left\|e_{k}(t)\right\| \leq \sqrt{\frac{\alpha_{2} \bar{\alpha}_{1}}{\alpha_{1}\left(\bar{\alpha}_{1}-\sum_{2}^{r} \bar{\alpha}_{j}\right)}} \sup _{k}\left\|e_{k}(0)\right\|, \quad \text { and } \\
\sup _{k}\left\|V_{k}(t)\right\|_{p} \leq \frac{\bar{\alpha}_{1}}{\bar{\alpha}_{1}-\sum_{2}^{r} \bar{\alpha}_{j} \sup _{k} V_{k}(0),} \\
\sup _{k}\left\|e_{k}(t)\right\|_{2 p} \leq \sqrt{\frac{\alpha_{2} \bar{\alpha}_{1}}{\alpha_{1}\left(\bar{\alpha}_{1}-\sum_{2}^{r} \bar{\alpha}_{j}\right)}} \sup _{k}\left\|e_{k}(0)\right\| .
\end{aligned}
$$


Let us define $z_{N}(t)=(1 / N) \sum_{1}^{N} e_{k}(t)$. This implies that $e_{N}(0)=$ $N z_{N}(0)-(N-1) z_{N-1}(0)$. Pick some $q>1$. If $\left\|\bar{x}_{N}(0)-w(0)\right\| \leq\left(M / N^{q}\right)$ for some $\mathrm{M}>0$, then $\left\|\left(e_{N}(0) / N\right)\right\| \leq\left(2 M / N^{q}\right)$. Therefore, $V_{N}(0) \leq$ $\left(4 M^{2} \alpha_{2} / N^{2 q-2}\right)$.

From the $\mathrm{I} / \mathrm{O}$ properties of discrete LTI systems, it follows that if $V_{N}(0) \leq\left(M_{1} / N^{2 q-2}\right)$, for some $M_{1}>0, q>1$, it follows that for some $M_{2}, M_{3}>0$,

$$
\sup _{t \geq 0} V_{N}(t) \leq \frac{M_{2}}{N^{2 q-2}}
$$

and

$$
\left\|V_{N}(t)\right\|_{p} \leq \frac{M_{3}}{N^{2 q-2}}
$$

Therefore, it follows that both $\sup _{t \geq 0}\left\|e_{N}(t)\right\|,\left\|e_{N}(t)\right\|_{2 p}$ are bounded by some sequence of the form $\left(M^{*} / N^{q-1}\right)$ for some $q>1$.

Therefore, $w(t)$ is a meaningful average.

Now, we will show that if $w(t)$ is a meaningful average in the strong sense, then it satisfies the differential equation, $\dot{w}=f(w, w, \ldots, w, u)$. If $w(t)$ is a meaningful average in the strong sense, consider the solution of the string corresponding to the initial condition sequence, $\{w(0)\}$. The corresponding solution for the string is the sequence, $\{x(t)\}$, where $\dot{x}=f(x, x, \ldots, x, u)$ and $x(0)=w(0)$. From the definition of a meaningful average, it follows that for some $\bar{M}, \beta>0, \| x(t)-$ $w(t) \| \leq\left(\bar{M} / N^{\beta}\right)$ for all $N$. Since the left hand side of the inequality is independent of $N$, it follows that $x(t) \equiv w(t)$ for all instants of time. Therefore, $w(t)$ satisfies the differential equation.

Remark Notice the equivalence of $\mathcal{L}_{p}$, averages, since the proof does not really depend upon $p$, as long as $p \geq 2$. This is important, since we now know that a solution to

$$
\dot{w}=f(w, w, \ldots, w, u)
$$

is a meaningful average in any $\mathcal{L}_{p}$ sense.

We will now deal with a collection of systems that have the same state space dimension, but not necessarily identical dynamics.

$$
\dot{x}_{i}=f_{i}\left(x_{i}, x_{i-1}, x_{i-2}, \ldots, x_{i-r+1}, u(t)\right), \quad i=1,2, \ldots
$$

We are interested in a construction of an ensemble average corresponding to the case when all initial conditions converge to $w_{0}$. 
THeOREM 3.2 We make the following assumptions as a first step towards analyzing the above system of differential equations:

- The vector fields, $f_{i}, i=1,2, \ldots$ are smooth and furthermore, they converge uniformly to a smooth vector field, $g$ on every compact set $K$ in $\underbrace{\Re^{n} \times \Re^{n} \times \cdots \times \Re^{n} \times \Re^{m}}$ and that $\left\|f_{k}-g\right\|_{\infty} \leq M$ for some $M>0$ and for all its arguments.

- The vector field $g$ satisfies the conditions 1,2 , and 3 of Theorem 3.1. Then, the solutions of

$$
\dot{x}_{i}=f_{i}\left(x_{i}, x_{i-1} \ldots, x_{i-r+1}, u\right),
$$

and

$$
\dot{w}=g(w, w, \ldots, w, u),
$$

are such that

- $\left\|x_{k}(t)-w(t)\right\|_{\infty}$ is uniformly bounded in $k$ and $\lim _{k \rightarrow \infty} \| x_{k}(t)-$ $w(t) \|_{\infty}=0$, if $\lim _{k \rightarrow \infty}\left\|x_{k}(0)-w(0)\right\|=0$.

Proof We will define $\mathrm{e}_{k}(t):=x_{k}(t)-w(t)$. From the previous theorem, there exists a sequence of Lyapunov functions, for the following string

$$
\dot{x}_{i}=g\left(x_{i}, x_{i-1}, \ldots, x_{i-r+1}, u\right), \quad i=1,2, \ldots,
$$

such that

$$
\dot{V}_{k} \leq-\bar{\alpha}_{1} V_{k}+\sum_{j=2}^{r} \bar{\alpha}_{j} V_{k-j+1} .
$$

For the string under consideration, differentiation of the Lyapunov functions yields:

$$
\begin{aligned}
\dot{V}_{k}= & \frac{\partial V_{k}}{\partial t}+\frac{\partial V_{k}}{\partial e_{k}} \dot{e}_{k} \\
= & \frac{\partial V_{k}}{\partial t}+\frac{\partial V_{k}}{\partial e_{k}}\left[f_{k}\left(e_{k}+w, e_{k-1}+w, \ldots, e_{k-r+1}+w, u\right)\right. \\
& \quad-g(w, w, w, \ldots, w, u)] \\
= & \frac{\partial V_{k}}{\partial t}+\frac{\partial V_{k}}{\partial e_{k}}\left[g\left(e_{k}+w, e_{k-1}+w, \ldots, e_{k-r+1}+w, u\right)-g(w, w, \ldots, w, u)\right] \\
+ & \frac{\partial V_{k}}{\partial e_{k}}\left[f_{k}\left(e_{k}+w, \ldots, e_{k-r+1}+w, u\right)\right. \\
& \left.\quad-g\left(e_{k}+w, e_{k-1}+w, \ldots, e_{k-r+1}+w, u\right)\right] \\
& \leq-\bar{\alpha}_{1} V_{k}+\sum_{j=2}^{r} \alpha_{j} V_{k-j+1}+\alpha_{4} M \sqrt{\frac{V_{k}}{\alpha_{1}}} .
\end{aligned}
$$


CLAIM If $\sup _{k}\left\|V_{k}(0)\right\|<M_{1}$, where $M_{1}>\left(\alpha_{4}^{2} M^{2} / \alpha_{1}\left(\bar{\alpha}_{1}-\sum_{2}^{r} \bar{\alpha}_{j}\right)\right)$, then $V_{k}(t)<M_{1}$ for all $k$ and for all instants of time t. Furthermore, if $\left\|V_{k}(0)\right\| \rightarrow 0$ as $k \rightarrow \infty$, then $\lim _{k \rightarrow \infty} \sup _{t \geq 0}\left\|e_{k}(t)\right\|=0$.

Proof of Claim Otherwise, pick the smallest $K$ such that $\sup _{t \geq 0}\left\|V_{K}(t)\right\|>M_{1}$. Note that on the surface, $V_{K}=M_{1}, \dot{V}_{K}<0$, provided $V_{j} \leq M_{1}, 0 \leq j \leq K$, which is the case here. Consequently, if $V_{k}(0)<M_{1}$ it must follow that $V_{k}(t)<M_{1}$. This is a contradiction.

Since, we have just proven that $\left\|e_{k}(t)\right\|$ is uniformly bounded, every $e_{k}(t)$ is contained in the same compact set in $\Re^{n}$. Since the vector fields, $f_{k}$ converge to $g$ uniformly on every compact set, it follows that $m_{k}:=\sup _{k}\left\|f_{k}-g\right\|$ goes to zero as $k \rightarrow \infty$. Consequently, the equation for $\dot{V}_{k}$ can be rewritten as

$$
\begin{gathered}
\dot{V}_{k} \leq-\bar{\alpha}_{1} V_{k}+\sum_{2}^{r} \bar{\alpha}_{j} V_{k-j+1}+\alpha_{4} M_{1} m_{k}, \\
\Rightarrow\left\|V_{k}(t)\right\|_{\infty} \leq \sum_{j=2}^{r} \frac{\bar{\alpha}_{j}}{\bar{\alpha}_{1}}\left\|V_{k-j+1}\right\|_{\infty}+V_{k}(0)+\frac{\alpha_{1}}{\bar{\alpha}_{1}} M_{1} m_{k} .
\end{gathered}
$$

From the comparison principle [15] and the properties of stable discrete filters with decaying inputs, it follows that $\lim _{k \rightarrow \infty}\left\|V_{k}(t)\right\|_{\infty}=0$. Consequently, $\lim _{k \rightarrow \infty}\left\|e_{k}(t)\right\|_{\infty}=0$.

Remark Clearly, the ensemble average for this collection of dynamical systems and for this set of initial conditions is $w(t)$. Since, $f_{\mathrm{i}}$ 's may deviate significantly from $g$, it is difficult to establish that $w(t)$ is a meaningful average for the collection of dynamical systems.

\section{CONCLUSIONS}

In this paper, we have considered two countable collections of dynamical systems - in the first collection, all constituent systems have identical dynamics, while in the other collection, there is a limit for the dynamics of its constituents. For the first collection, we have shown, under the conditions of stability of individual responses and the weakness of interaction among systems, there is a meaningful average that is a solution of finite dimensional ordinary differntial equation. For the second collection, the result is as follows - if one considers a countable collection of dynamical systems having the 
limiting dynamics, similar to the first collection, and if a meaningful average can be constructed for such a system, then an ensemble average can be constructed for the second collection, if it has a limit for the sequence of initial conditions.

\section{Acknowledgements}

The authors thank the National Science Foundation and the Interdisciplinary Research Initiative of Texas A\&M University for providing financial support for this research.

\section{References}

[1] Aoki, M. (1968). "Control of Large-Scale Dynamic Systems by Aggregation", IEEE Transactions on Automatic Control, 13(3), 246-253.

[2] Arley, N. and Borchsenius, V. (1945). "On the theory of infinite systems of differential equations and their application to the theory of stochastic processes and the perturbation theory of quantum mechanics", Acta Mathematica, 76, 261-322.

[3] Bellman, R. (1947). "The boundedness of solutions of infinite systems of linear differential equations", Duke Mathematical Journal, 3, 695-706.

[4] Chu, K. C., "Decentralized control of high speed vehicle strings", Transportation Science, pp. 361 -383, June, 1974.

[5] Desoer, C. A. and Vidyasagar, M., "Feedback Systems: Input-Output Properties", Academic Press, New York, 1975.

[6] Golub, G. and Van Loan, C., "Matrix Computations", Second edition, The Johns Hopkins University Press, Baltimore, 1993.

[7] Ikeda, M., Siljak, D. D. and White, D. E., "An Inclusion Principle for Dynamic Systems", IEEE Transactions on Automatic Control, AC-29(3), March, 1984.

[8] Leontief, W. (1936). "Composite Commodities and the problem of Index Numbers", Econometrica, 4, 39-59.

[9] Levinson, N. (1946). "The asymptotic behavior of a system of linear differential equations", American Journal of Mathematics, 68, 1-6.

[10] Liapunov, A. M. and Fuller, A. T. (translator), "On the stability of motion", reprinted in the International Journal of Control, March, 1992.

[11] Rudin, W. (1976). "Principles of Mathematical Analysis", McGraw-Hill Book Company.

[12] Sezer, M. E. and Siljak, D. D., "Validation of Reduced-Order Models for Control Systems Design", Journal of Guidance, 5(5), 430-437, September-October, 1982.

[13] Shaw, L. (1972). "Existence and approximation of solutions to an infinite set of linear time invariant differential equations", SIAM Journal of Applied Mathematics, 22, 266-279.

[14] Simon, H. A. and Ando, A., "Aggregation of Variables in dynamic systems", Econometrica, 29, 111 - 38, April, 1961.

[15] Siljak, D., "Large-Scale Dynamic Systems: Stability and Structure", NorthHolland, New York, 1978.

[16] Siljak, D. (1991). "Decentralized Control of Complex Systems", Academic Press, 1991. 
[17] Stilwell, D. J. and Bishop, B. E., "Platoons of Underwater Vehicles: Communication, Feedback and Decentralized Control", IEEE Control Systems Magazine, pp. 45-52, December, 2000.

[18] Swaroop, D., "String stability of Interconnected Systems: An application to platooning in Automated Highway Systems", Ph.D. Dissertation, University of California, Berkeley, 1994.

[19] Swaroop, D., Hedrick, J. K., Chien, C. C. and Ioannou, P. (1994). "A comparison of spacing and headway control strategy for automatically controlled vehicles", Vehicle System Dynamics, 23(8), 597-625.

[20] Swaroop, D. and Hedrick, J. K., "String Stability of Interconnected Systems", IEEE Transactions on Automatic Control, pp. 349-357, March, 1996.

[21] Swaroop, D. and Rajagopal, K. R., "Intelligent Cruise Control Systems and Traffic Flow Stability", Transportation Research Journal, C(7), 329-352, December, 1999.

[22] Swaroop, D. and Rajagopal, K. R., "Aggregation of a class of interconnected, linear dynamical systems", accepted for publication in the Systems and Control Letters, September, 2000.

[23] Wang, P. K. C., Hadaegh, F. Y. and Lau, K., "Synchronized Guidance formation and attitude control of multiple free flying spacecraft", Journal of Guidance, Control and Dynamics, 22(1), 28-35 January-February, 1999. 


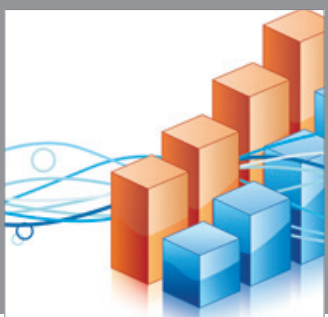

Advances in

Operations Research

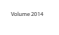

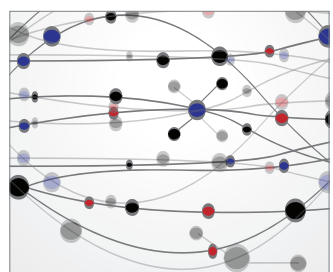

\section{The Scientific} World Journal
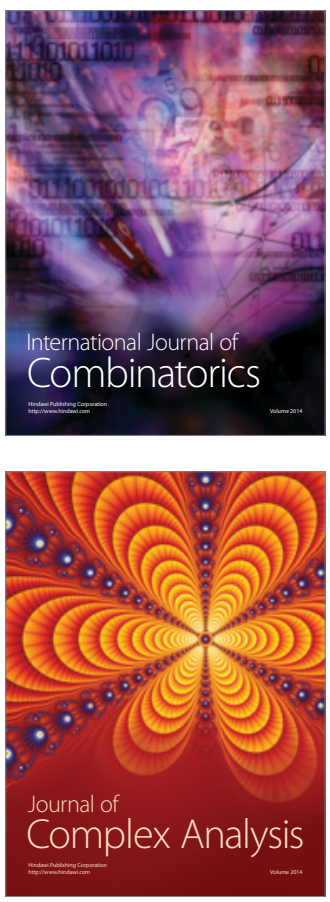

International Journal of

Mathematics and

Mathematical

Sciences
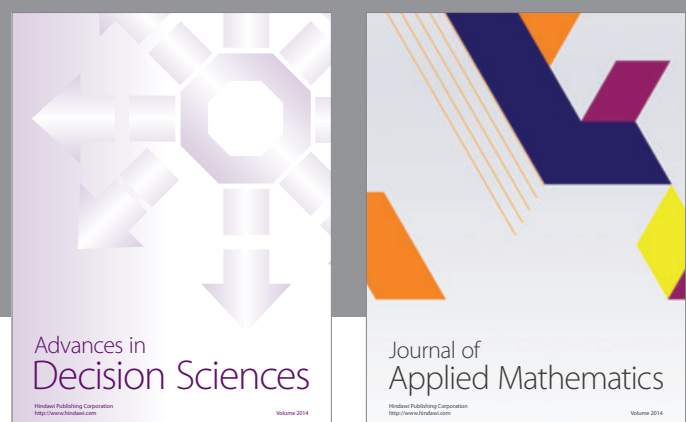

Journal of

Applied Mathematics
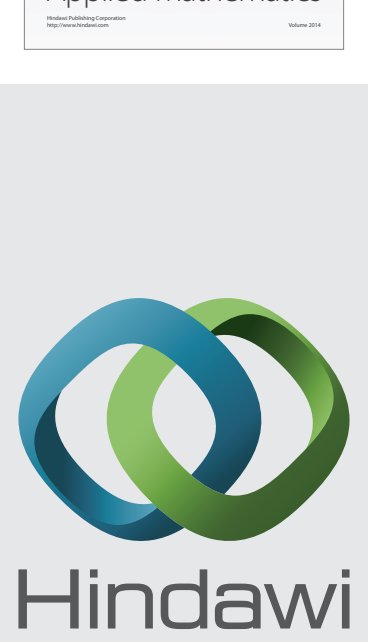

Submit your manuscripts at http://www.hindawi.com
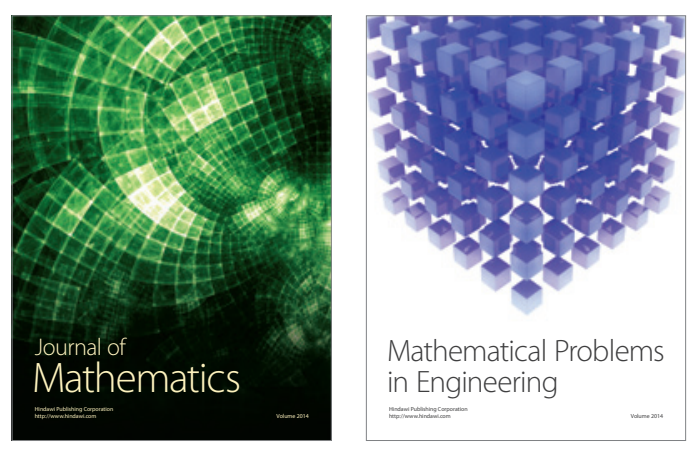

Mathematical Problems in Engineering
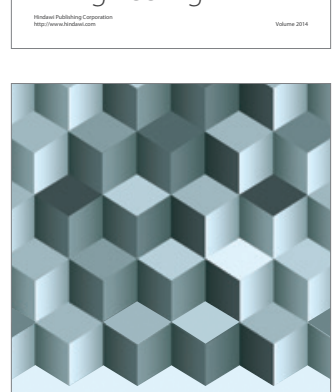

Journal of

Function Spaces
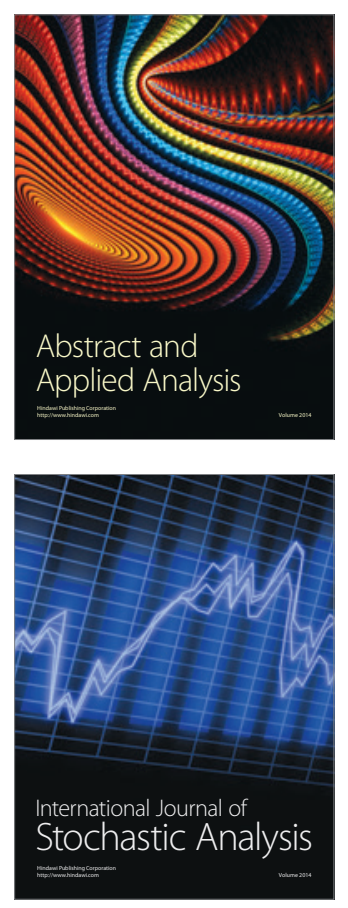

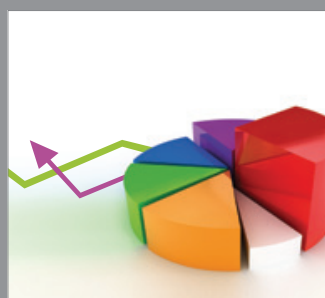

ournal of

Probability and Statistics

Promensencen
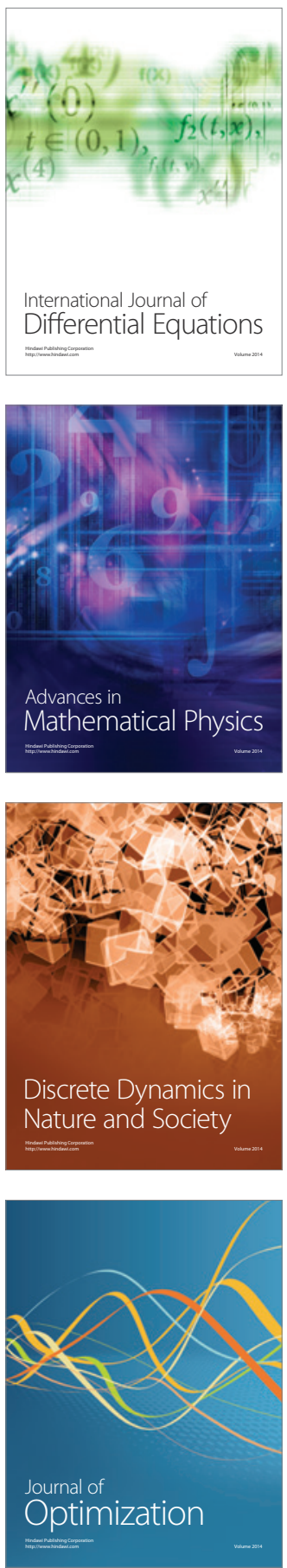